\title{
IPEC2005
}

\section{The 7th International} Power Engineering conference

\section{ABSTRASTS \& POGRAM}

29 November - 2 December 2005 MARINA MANDARIN HOTEL Singapore 
Abstract--This paper proposes a new control strategy for three-phase shunt active power filter that is capable to operate under three-wire or four-wire systems. It is based on Source Instantaneous Power. The principle of this control method is to eliminate undesired source currents. Calculation of source instantaneous power is needed to determine instantaneous power component that may not be created by the source. This calculation only needs source currents and phase information so the amount of sensors can be minimized. This control method can force source currents nearly sinusoidal and have unity power factor. Application in four-wire system will reduce neutral current at source side. Simulation is done to verify analysis.

Index Terms - active power filter (APF), instantaneous real power, instantaneous reactive power, 